\title{
LOFAR tied-array imaging and spectroscopy of solar S bursts ${ }^{\star}$
}

\author{
D. E. Morosan ${ }^{1}$, P. T. Gallagher ${ }^{1}$, P. Zucca ${ }^{1}$, A. O'Flannagain ${ }^{1}$, R. Fallows ${ }^{2}$, H. Reid ${ }^{3}$, J. Magdalenić ${ }^{4}$, G. Mann ${ }^{5}$,
} M. M. Bisi ${ }^{6}$, A. Kerdraon ${ }^{7}$, A. A. Konovalenko ${ }^{8}$, A. L. MacKinnon ${ }^{3}$, H. O. Rucker ${ }^{9}$, B. Thidé ${ }^{10}$, C. Vocks $^{5}$, A. Alexov ${ }^{11}$, J. Anderson ${ }^{12}$, A. Asgekar ${ }^{2,13}$, I. M. Avruch ${ }^{14,15}$, M. J. Bentum ${ }^{2,16}$, G. Bernardi ${ }^{17}$, A. Bonafede ${ }^{18}$, F. Breitling 5 , J. W. Broderick ${ }^{19,20}$, W. N. Brouw ${ }^{2,15}$, H. R. Butcher ${ }^{21}$, B. Ciardi ${ }^{22}$, E. de Geus ${ }^{2,23}$, J. Eislöffel ${ }^{24}$, H. Falcke ${ }^{25,2}$, W. Frieswijk ${ }^{2}$, M. A. Garrett ${ }^{2} 26$, J. Grießmeier ${ }^{27,28}$, A. W. Gunst ${ }^{2}$, J. W. T. Hessels ${ }^{2,29}$, M. Hoeft ${ }^{24}$, A. Karastergiou ${ }^{19}$, V. I. Kondratiev ${ }^{2,30}$, G. Kuper ${ }^{2}$, J. van Leeuwen ${ }^{2,29}$, D. McKay-Bukowski ${ }^{31,32}$, J. P. McKean ${ }^{2,15}$, H. Munk ${ }^{2}$, E. Orru², H. Paas ${ }^{33}$, R. Pizzo ${ }^{2}$, A. G. Polatidis ${ }^{2}$, A. M. M. Scaife ${ }^{20}$, J. Sluman ${ }^{2}$, C. Tasse ${ }^{7}$, M. C. Toribio ${ }^{2}$, R. Vermeulen ${ }^{2}$, and P. Zarka ${ }^{7}$

(Affiliations can be found after the references)

Received 10 March 2015 / Accepted 19 June 2015

\begin{abstract}
Context. The Sun is an active source of radio emission that is often associated with energetic phenomena ranging from nanoflares to coronal mass ejections (CMEs). At low radio frequencies $(<100 \mathrm{MHz})$, numerous millisecond duration radio bursts have been reported, such as radio spikes or solar S bursts (where S stands for short). To date, these have neither been studied extensively nor imaged because of the instrumental limitations of previous radio telescopes.

Aims. Here, LOw Frequency ARray (LOFAR) observations were used to study the spectral and spatial characteristics of a multitude of S bursts, as well as their origin and possible emission mechanisms.

Methods. We used 170 simultaneous tied-array beams for spectroscopy and imaging of S bursts. Since S bursts have short timescales and fine frequency structures, high cadence $(\sim 50 \mathrm{~ms})$ tied-array images were used instead of standard interferometric imaging, that is currently limited to one image per second.

Results. On 9 July 2013, over $3000 \mathrm{~S}$ bursts were observed over a time period of $\sim 8 \mathrm{~h}$. S bursts were found to appear as groups of short-lived (<1 s) and narrow-bandwidth $(\sim 2.5 \mathrm{MHz})$ features, the majority drifting at $\sim 3.5 \mathrm{MHz} \mathrm{s}^{-1}$ and a wide range of circular polarisation degrees $(2-8$ times more polarised than the accompanying Type III bursts). Extrapolation of the photospheric magnetic field using the potential field source surface (PFSS) model suggests that $\mathrm{S}$ bursts are associated with a trans-equatorial loop system that connects an active region in the southern hemisphere to a bipolar region of plage in the northern hemisphere.

Conclusions. We have identified polarised, short-lived solar radio bursts that have never been imaged before. They are observed at a height and frequency range where plasma emission is the dominant emission mechanism, however, they possess some of the characteristics of electroncyclotron maser emission.
\end{abstract}

Key words. Sun: corona - Sun: radio radiation - Sun: particle emission - Sun: magnetic fields

\section{Introduction}

The Sun is an active star that produces numerous large scale energetic phenomena often accompanied by solar radio bursts such as the Type I, II, III, IV and V radio bursts. At decimetre/metre wavelengths, numerous other radio bursts have been observed, for example metric spikes (Benz et al. 1982, 1996), drifting spikes (Elgaroy \& Sveen 1979) and supershort solar radio bursts (Magdalenić et al. 2006). These bursts exhibit a variety of fine frequency structures and timescales of $<1 \mathrm{~s}$, which can be an indicator of small scale processes occurring in the solar corona. However, at longer wavelengths, corresponding to frequencies $<100 \mathrm{MHz}$, there have been very few studies of short fine structure radio bursts due to sensitivity and imaging limitations of previous radio telescopes.

At low frequencies, below $100 \mathrm{MHz}$, Ellis (1969) used 32 broad-band dipoles to identify a new type of short duration radio burst that he named fast drift storm bursts. Later, McConnell (1982) observed these bursts using the Llanherne

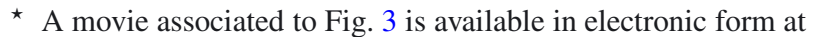
http: //www . aanda.org radio telescope consisting of 4096 dipoles (Ferris et al. 1980). He showed that their drift rate is slower than that of Type III radio bursts (about $1 / 3$ the drift rate of Type IIIs). McConnell (1982) renamed fast drift storms as "solar S bursts" due to their similarity to jovian $\mathrm{S}$ bursts. $\mathrm{S}$ bursts appear as narrow drifting lines in dynamic spectra with a temporal width of up to a few tens of milliseconds and total duration of about $1 \mathrm{~s}$. They have been observed in a frequency range of 30-150 MHz (Ellis 1982; McConnell 1982). They have short instantaneous bandwidths of about $120 \mathrm{kHz}$ and instantaneous duration of $50 \mathrm{~ms}$ at frequencies of $40 \mathrm{MHz}$ (McConnell 1983). More recently, Briand et al. (2008) and Melnik et al. (2010) have reported observations of $\mathrm{S}$ bursts in the frequency range 10-30 MHz. These were observed with the UTR-2 radio telescope consisting of over 2000 dipoles with a total area of over $30000 \mathrm{~m}^{2}$ (Abranin et al. 2001; Melnik et al. 2011). Most S bursts have negative drift rates, i.e. from high to low frequencies, however a few $\mathrm{S}$ bursts have positive drift rates (McConnell 1982; Briand et al. 2008). All $\mathrm{S}$ bursts occurred during times of other solar activity such as Type III and Type IIIb radio bursts (McConnell 1982; Briand et al. 2008; Melnik et al. 2010). The majority of S burst studies 


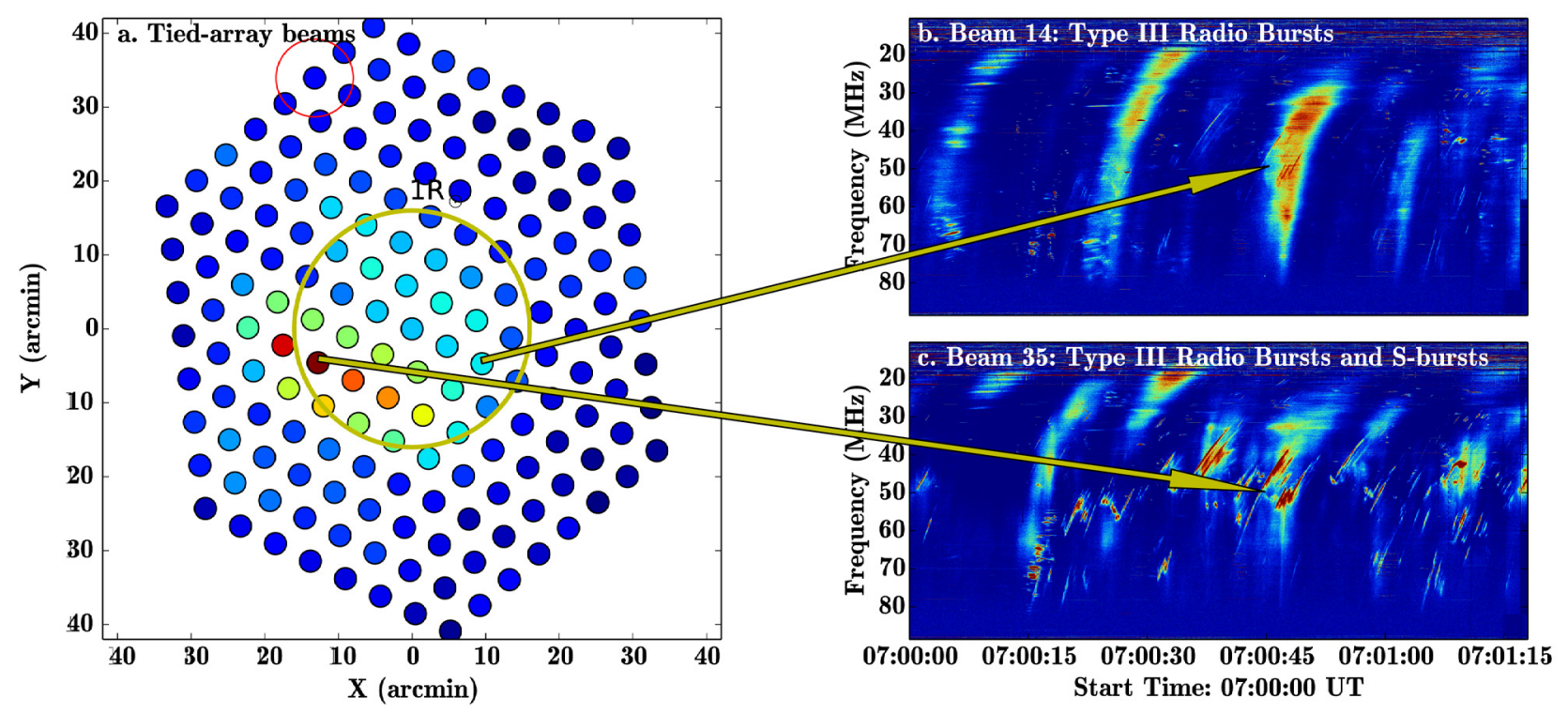

Fig. 1. LOFAR tied-array beam observations of Type III radio bursts and solar S bursts. a) Map of 170 tied-array beams covering a field-of-view of $\sim 1.3^{\circ}$ about the Sun. The full-width half-maximum (FWHM) of the beams at zenith at a frequency of $60 \mathrm{MHz}$ is represented by the red circle and the size of the optical Sun is represented by the yellow circle. b) Dynamic spectrum recorded for a period of 1.3 min corresponding to Beam 14 containing Type III radio bursts. c) Dynamic spectrum recorded for a period of 1.3 min corresponding to Beam 35 containing Type III radio bursts and $\mathrm{S}$ bursts. The two arrows indicate the beams that recorded the two dynamic spectra in $\mathbf{b}$ ) and $\mathbf{c}$ ) pointing at the time and frequency corresponding to the intensity values in a).

consider plasma emission as the most probable emission mechanism (Zaitsev \& Zlotnik 1986; Melnik et al. 2010). To date, $\mathrm{S}$ bursts have only been observed with highly sensitive instruments as they have a low intensity compared to the majority of radio bursts. So far, there has been no imaging of $S$ bursts for more detailed studies of their origins and emission mechanisms.

With the recent development of the LOw Frequency ARray (LOFAR, van Haarlem et al. 2013), we now have the possibility to observe solar $\mathrm{S}$ bursts in high time and frequency resolution dynamic spectra and for the first time obtain spatial characteristics of $\mathrm{S}$ bursts from imaging. In this paper, LOFAR tied-array beams were used to study the spatial and spectral characteristics of S bursts. In Sect. 2 we give an overview of the LOFAR instrument and the observational method. In Sect. 3 we present the results of the spectral and imagining analysis which are discussed in Sect. 4.

\section{Observations and data analysis}

On 9 July 2013 starting at 07:00 UT, over $3000 \mathrm{~S}$ bursts were observed during a LOFAR observation campaign that lasted until 14:30 UT. These observations were made at times of medium solar activity in the form of three C-class flares and the presence of four $\beta \gamma \delta$ active regions (NOAA 11784, 11785, 11787 , 11789) on the visible side of the solar disc. In this time interval, a long-lived noise storm was observed above active region NOAA 11785 at frequencies greater than $150 \mathrm{MHz}$.

LOFAR represents a new milestone in low radio frequency arrays and was constructed by the Netherlands Institute for Radio Astronomy (ASTRON). It consists of $\sim 7000$ antennas: Low Band Antennas (LBAs) operating at frequencies of 10-90 MHz and High Band Antennas (HBAs) which operate at $110-240 \mathrm{MHz}$ (van Haarlem et al. 2013). These antennas are distributed in 24 core stations and 14 remote stations across the Netherlands and 9 international stations across Europe.

In this paper, we used one of LOFAR's beam formed modes (Stappers et al. 2011; van Haarlem et al. 2013) in the LBA frequency range. Figure 1a shows 170 simultaneous beams which were used to observe the Sun covering a field-of-view of $1.3^{\circ}$ centred on the Sun. The 24 LOFAR core stations were used to produce these beams. Each beam produces a high time and frequency resolution dynamic spectra $(\sim 10 \mathrm{~ms} ; 12.5 \mathrm{kHz})$ as can be seen in Figs. $1 \mathrm{~b}$ and c, in which the radio bursts can be identified. Since each beam has a spatial location, it can be used to extract the intensity of the radiation at that specific location. These intensity values can be plotted as "macro-pixels" onto the tied array map for a chosen time and frequency as seen in Fig. 1a. The arrows in Fig. 1 denote the time, frequency and beam where the intensity value was extracted.

Radio sources can be imaged by interpolating between the intensity of radiation from all beams and producing tied-array images of radio bursts (for more details, see Morosan et al. 2014). All images of S bursts were averaged over a frequency bin of $1 \mathrm{MHz}$ to reduce spectral noise and over a time period of $50 \mathrm{~ms}$. Side lobes are often visible in tied-array images due to the coherent combination of tied-array beams, however, most side lobes have been excluded manually as they occur at the same time as the source and always at the same location some distance away from the source. Due to the size of tied-array beams there is an uncertainty in determining the exact location of the radio source as the source could be located anywhere within the beam. This uncertainty is estimated to be $0.2 R_{\odot}$ at $80 \mathrm{MHz}$ and $0.6 R_{\odot}$ at $30 \mathrm{MHz}$ and it corresponds to the half power beam width. The main source of uncertainty in beam pointing is the ionosphere, which is most pronounced during periods of elevated solar activity. During our observations, there was no significant X-ray activity other than a few C-class flares. In addition, the centroid position of our sources was found not to vary significantly over many hours.

\section{Results}

\section{1. $S$ bursts spectral characteristics}

An example of $\mathrm{S}$ bursts that occurred during a time period of 1.3 min on 9 July 2013 at 07:00 UT is shown in Fig. 1c. The 
D. E. Morosan et al.: LOFAR tied-array imaging and spectroscopy of solar S bursts
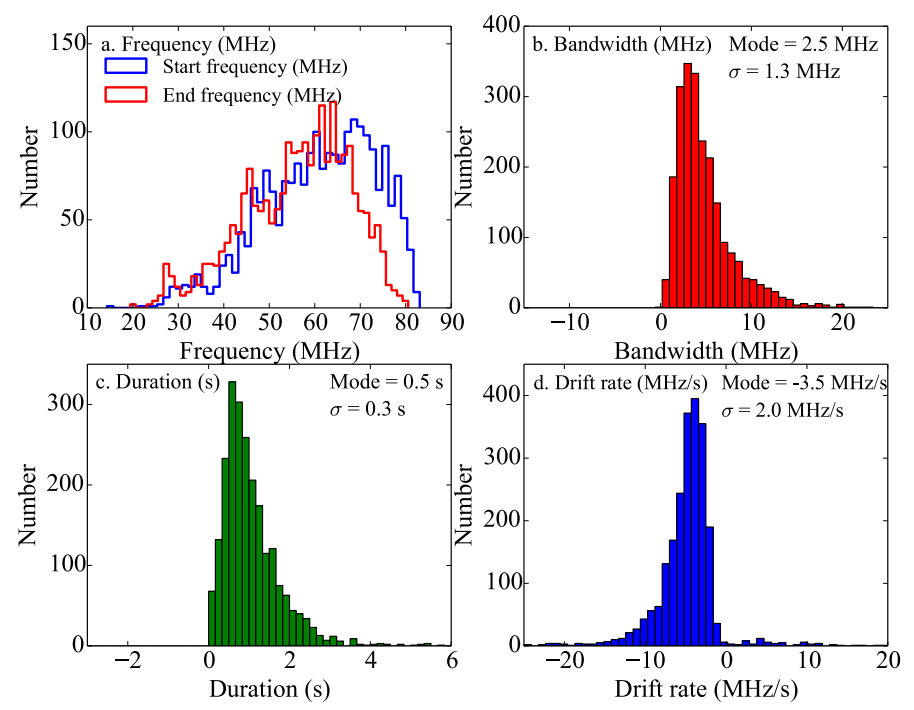

Fig. 2. S-burst property distributions: start and end frequency a), total frequency bandwidth b), duration c) and drift rate d). The most frequently occurring value (mode) and the standard deviation are shown. The standard deviation was calculated using a normal distribution fit to each of the distributions.

S bursts were recorded simultaneously with other solar activity mainly in the form of Type III and Type IIIb radio bursts, some of which did not originate at the same location as the $\mathrm{S}$ bursts as can be seen in Figs. $1 b$ and c.

Over $3000 \mathrm{~S}$ bursts have been recorded during a time interval of $8 \mathrm{~h}$ on the same day in the LBA frequency range. Due to a variety of shapes (various lengths, time profiles, frequency profiles) and various intensity levels, similar to Type III/Type IIIb bursts, it was necessary to identify these bursts manually. Distributions of their characteristics such as the start and end frequency, total bandwidth, duration and drift rate are shown in Fig. 2. These distributions are asymmetrical and therefore the mode of the distributions is shown instead of the mean due to the fact that they are skewed. Most $\mathrm{S}$ bursts had short durations of $<1 \mathrm{~s}$ and instantaneous durations at a fixed frequency of $<400 \mathrm{~ms}$, some as small as $20 \mathrm{~ms}$. They were observed at frequencies between 20 to $80 \mathrm{MHz}$ and the majority had a total bandwidth of $\sim 2.5 \pm$ $1.3 \mathrm{MHz}$ and instantaneous bandwidths at a fixed time varying between $0.1-1.5 \mathrm{MHz}$. Very few $\mathrm{S}$ bursts had longer bandwidths $>20 \mathrm{MHz}$. Their drift rates varied with frequency from $-1 \mathrm{MHz} \mathrm{s}^{-1}$ at frequencies of $20 \mathrm{MHz}$ to $-7 \mathrm{MHz} \mathrm{s}^{-1}$ at $75 \mathrm{MHz}$ (Fig. 2) but most bursts had a drift rate of $-3.5 \pm 2.0 \mathrm{MHz} \mathrm{s}^{-1}$. The standard deviation of all distributions is a significant fraction of the mode, therefore there is a wide spread in duration, bandwidth and drift rate of $\mathrm{S}$ bursts.

Table 1 shows that these values are comparable to the findings of McConnell (1982, 1983). A few S bursts were found to have positive drift rates and an example is shown in Fig. 3a. Positive drifting $\mathrm{S}$ bursts have also been reported before by McConnell (1982, 1983), Briand et al. (2008), Melnik et al. (2010).

Full Stokes data were recorded starting at 11:40:00 UT for a duration of $1 \mathrm{~h}$. During this time several hundred S bursts were observed as well as Type III radio bursts. The observed data is uncalibrated and we cannot estimate the degree of polarisation accurately, however, we can compare the $\mathrm{S}$ bursts polarisation to the background Type III radio bursts. Type IIIs emitted at the harmonic of the local plasma frequency are believed to be weakly polarised ( $<10 \%$, Dulk \& Suzuki 1980). We have
Table 1. S bursts characteristics estimated from LOFAR dynamic spectra are compared to results by McConnell (1982).

\begin{tabular}{|c|c|c|}
\hline & LOFAR & McConnell \\
\hline Frequency range (MHz) & $20-80$ & $30-82$ \\
\hline Duration (s) & $0.5 \pm 0.3$ & 1.2 \\
\hline Instantaneous duration (ms) & $20-400$ & 50 \\
\hline Total bandwidth (MHz) & $2.5 \pm 1.3$ & $<5$ \\
\hline Instantaneous bandwidth (MHz) & $0.1-1.5$ & 0.1 \\
\hline Drift rate $\left(\mathrm{MHz} \mathrm{s}^{-1}\right)$ & $-3.5 \pm 2.0$ & -3 \\
\hline
\end{tabular}

found a wide range of different circular polarisation degrees for $\mathrm{S}$ bursts from 2-8 times more polarised than the accompanying Type III bursts. This agrees with previous studies in which the $\mathrm{S}$ bursts studied were found to have a strong component of circular polarisation (McConnell 1982).

\section{2. $S$ bursts spatial characteristics}

An example of a number of $\mathrm{S}$ bursts analysed in more detail is shown in Fig. 3. Figure 3 is a snapshot from the movie accompanying this paper. Figure 3 a shows a $4 \mathrm{~s}$ LOFAR dynamic spectrum containing $\mathrm{S}$ bursts with both negative and positive drift rates. Figures $3 b$ and $c$ show the location of these $S$ bursts overlaid on extreme ultraviolet images (EUV) at $171 \AA$ from the Atmospheric Imaging Assembly (AIA; Lemen et al. 2012) onboard NASA's Solar Dynamic Observatory (SDO). S bursts are imaged at frequencies of $64-65 \mathrm{MHz}$ to sample the negatively drifting $\mathrm{S}$ bursts and 51-52 MHz to sample the positively drifting $\mathrm{S}$ bursts.

Tied-array imaging can produce images at very high cadence (50 ms) which is necessary for radio bursts with durations of $<1 \mathrm{~s}$. However the beams have a FWHM of $12.6^{\prime}$ at a frequency of $50 \mathrm{MHz}$ as they can only be produced by the LOFAR core. Its $2 \mathrm{~km}$ baseline is too small to produce arcsecond beam sizes. As a result the source sizes appear large. We cannot detect any movement in the source centroids due to frequency change. Both negatively and positively drifting $S$ bursts appear to originate at the same location. All S bursts analysed in this observation campaign on 9 July 2013 appear to originate at the same location with insignificant deviations in the source centroids $\left(<0.1 R_{\odot}\right)$. It is possible that this results from the reduced spatial resolution of tied-array beams imaging.

We investigated the origin of these $\mathrm{S}$ bursts by relating the source locations to the coronal magnetic field. Figure 4 shows that the radio source does not seem to be directly located on top of known active regions but it appears to be close to the top of trans-equatorial loops connecting a complex active region in the southern hemisphere and a large area of bipolar plage in the northern hemisphere. The top of these equatorial loops is located at a distance of about $\sim 1.8 R_{\odot}$ from the solar centre. This distance is calculated from the 3D PFSS extrapolation of the loop and it represents the radial height of the loop top from the surface. The spatial coincidence of the $S$ bursts and the top of these equatorial loops in Fig. 4 suggests that $S$ bursts are associated with these loops.

\section{Discussion and conclusion}

Over $3000 \mathrm{~S}$ bursts were observed by LOFAR at times of medium solar activity and simultaneously with other radio emission such as Type III and Type IIIb bursts. Most $\mathrm{S}$ bursts had very short durations $(\sim 0.5 \mathrm{~s})$ and short bandwidths $(\sim 2.5 \mathrm{MHz})$. 

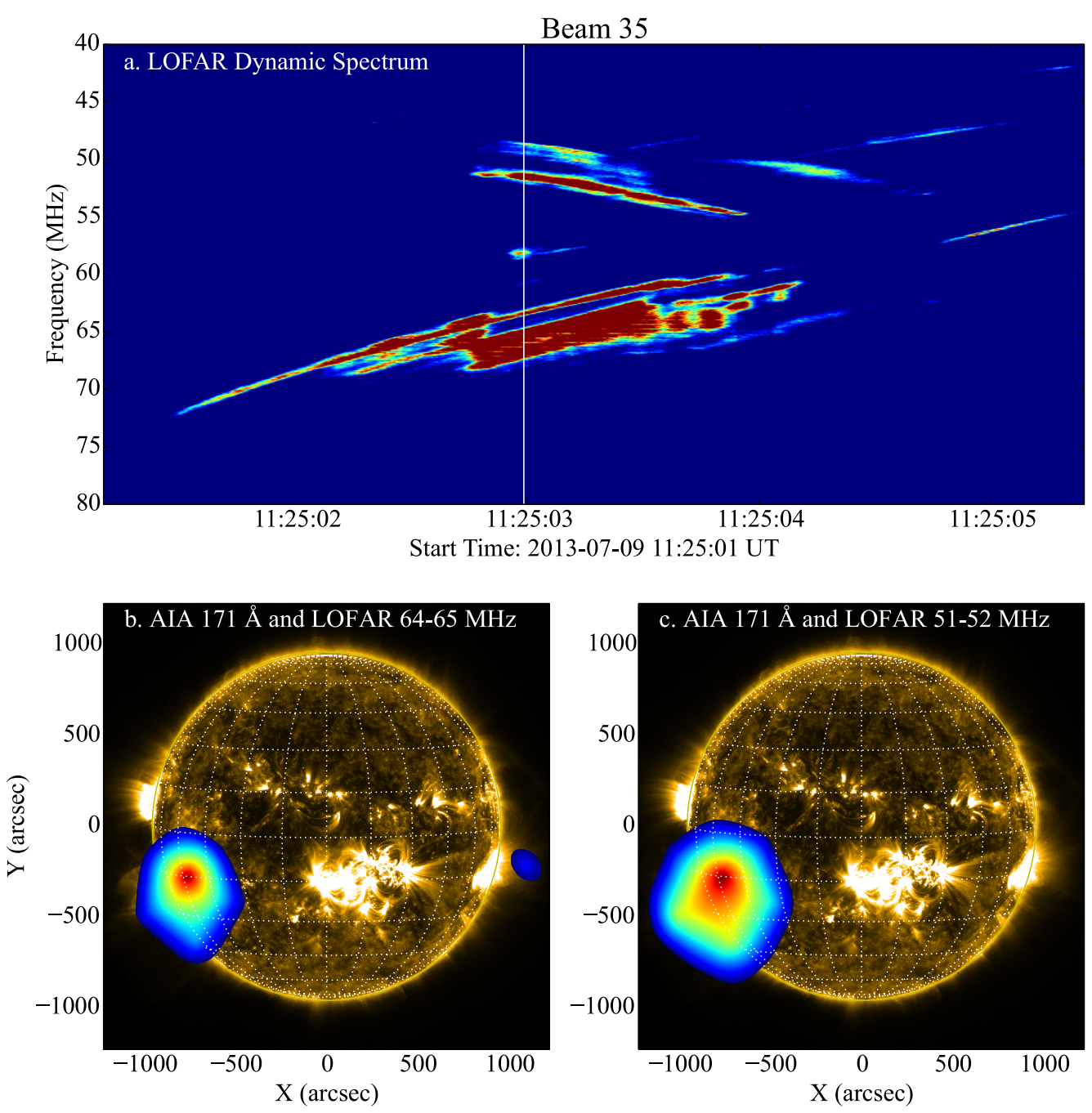

Fig. 3. Location of $\mathrm{S}$ bursts at two separate frequencies. a) LOFAR 4 s dynamic spectrum showing negatively and positively drifting $\mathrm{S}$ bursts. The white line marks the timestamp in the images below. b) SDO/AIA $171 \AA$ image at 11:25:11 UT and the overlaid LOFAR source of the negative drifting $S$ burst at a frequency of 64-65 MHz at the time marked by the white line in a). c) SDO/AIA $171 \AA$ image 11:25:11 UT and the overlaid LOFAR source of the positive drifting $\mathrm{S}$ bursts at a frequency of $51-52 \mathrm{MHz}$ at the time marked by the white line in a). An animation of the temporal evolution is available in the online edition.

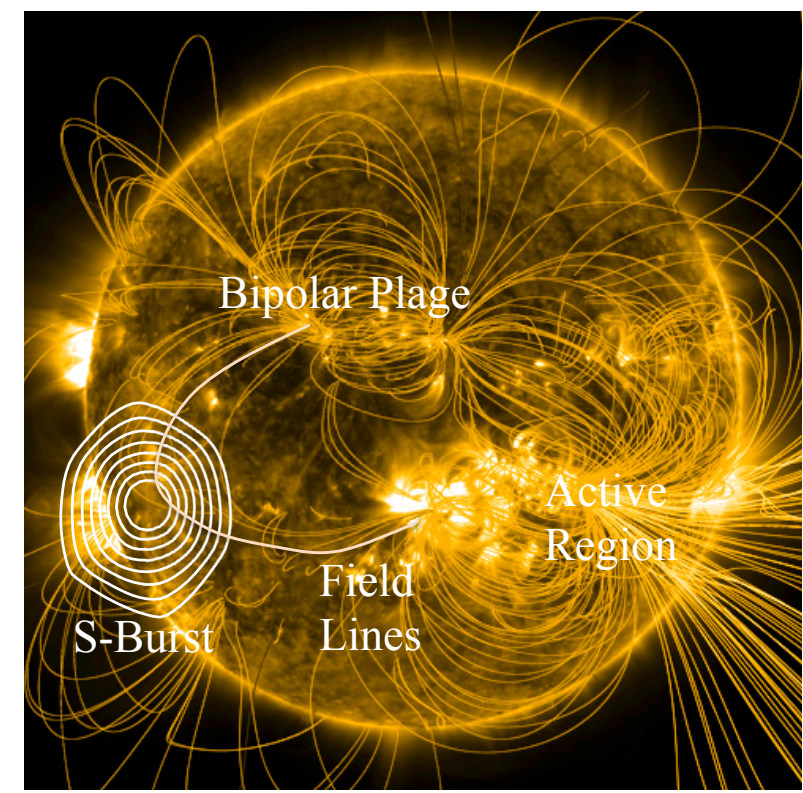

Fig. 4. $S$ bursts source position and their relation to the coronal magnetic field. The AIA $171 \AA$ image at 11:24:12 UT is overlaid with PFSS magnetic field extrapolations. The white contours represent the LOFAR S bursts source at 11:24:56 UT and a frequency of 74-75 MHz. One of the trans-equatorial loops connecting the bipolar page to the active region is labelled in the figure.
Their drift rates varied with frequency with the majority drifting at $-3.5 \mathrm{MHz} \mathrm{s}^{-1}$ at frequencies of $20-80 \mathrm{MHz}$ (Table 1) which is about $1 / 3$ of the Type III drift rate. Type IIIs drift at $-11.33 \mathrm{MHz} \mathrm{s}^{-1}$ in the frequency range of $40-70 \mathrm{MHz}$ according to Mann \& Klassen (2002). The majority of S bursts are found at frequencies between $60-70 \mathrm{MHz}$ (Fig. 2a), but this result may be influenced by the $60 \mathrm{MHz}$ peak in the sensitivity of LBAs antennas (van Haarlem et al. 2013). S bursts were found to be significantly more polarised than Type IIIs. Calibrated polarisation observations of $\mathrm{S}$ bursts will allow for these properties to be further studied in a follow on paper.

For the first time, we were able to image solar $\mathrm{S}$ bursts. Tiedarray imaging shows that all $\mathrm{S}$ bursts originate in the same spatial location with very little deviation of the source centroid. There seem to be no features in the solar corona at the location of $\mathrm{S}$ bursts sources other than long trans-equatorial loops connecting an active region in the southern hemisphere and an extended area of bipolar plage in the northern hemisphere. We have investigated two possible emission mechanisms responsible for solar $\mathrm{S}$ bursts: plasma emission and electron-cyclotron maser (ECM) emission which are discussed below.

So far, plasma emission has been proposed by Zaitsev \& Zlotnik (1986) and Melnik et al. (2010) as the mechanism responsible for emission of S bursts. Zaitsev \& Zlotnik (1986) suggest that electrons with velocities 10-20 times above their thermal velocity excite plasma waves near the upper hybrid resonance frequency. These waves are then scattered by ions 
producing electromagnetic waves at the plasma frequency $\left(f_{\mathrm{p}}\right)$. Melnik et al. (2010) propose a model in which S bursts are generated by the coalescence of magnetosonic waves and Langmuir waves and derive a minimum magnetic field of $2 \mathrm{G}$ necessary for the generation of these bursts at the plasma frequency $\left(f_{\mathrm{p}}\right)$.

In our observation, $\mathrm{S}$ bursts appear to be associated with equatorial loops stretching to high altitudes $\left(\sim 1.8 R_{\odot}\right)$ where plasma emission is likely the dominant emission mechanism (Gary \& Hurford 1989). Knowing the height of these loops, a density model can be used to find the electron density at this distance. Using the electron density we can estimate the frequency of plasma radiation at the top of the loops using the following equation:

$f=n f_{\mathrm{p}}=n C \sqrt{N_{\mathrm{e}}}$.

The emission frequency, $f$, is given by the local plasma frequency, $f_{\mathrm{p}}$, multiplied by the harmonic number $n$ and is directly proportional to the square root of the electron density, $N_{\mathrm{e}}$, in $\mathrm{cm}^{-3}$, where $C=8980 \mathrm{~Hz} \mathrm{~cm} \mathrm{~cm}^{3 / 2}$ is the constant of proportionality.

We used the radial electron density models of Newkirk (1961), Saito et al. (1977) and Mann et al. (1999) and the timedependent density model of Zucca et al. (2014) to estimate electron densities at specific distances. These models predict electron densities in the range of $1.2 \times 10^{6}-1.1 \times 10^{7} \mathrm{~cm}^{-3}$ at $\sim 1.8 R_{\odot}$. This corresponds to a frequency range of $\sim 10-30 \mathrm{MHz}$ for fundamental plasma emission and $\sim 20-60 \mathrm{MHz}$ for harmonic plasma emission, frequency ranges in which $\mathrm{S}$ bursts are found. In addition, $S$ bursts show a drift rate dependence with frequency similar to that of Type III radio bursts (McConnell 1980). A similar drift rate dependence with frequency was also found in our analysis which will be discussed in a follow-on paper. It is therefore possible that $\mathrm{S}$ bursts are a form of plasma emission. The high degree of polarisation of some of these bursts requires that they are emitted at the fundamental plasma frequency (Wentzel 1984).

However, $\mathrm{S}$ bursts have a significantly slower drift rate than Type III radio bursts (about $1 / 3$ of the Type III drift rate), very narrow bandwidths and short lifetimes and they are significantly more polarised. This is indicative of ECM emission. Jovian $\mathrm{S}$ bursts, which are similar in appearance to solar $\mathrm{S}$ bursts, originate due to cyclotron maser emission in the flux tubes connecting Io or Io's wake to Jupiter (Hess et al. 2007). For ECM to occur in the solar corona, the electron-cyclotron frequency ( $f_{B}=e B / 2 \pi m_{\mathrm{e}}$, where $B$ is the magnetic field) has to be greater than the plasma frequency (Melrose 1991). Therefore we require a high $B$ field at the emission site in the corona $(>20 \mathrm{G})$, as well as low electron plasma density $\left(N_{\mathrm{e}}\right)$. While the plasma density is low at large heights in the corona where $\mathrm{S}$ bursts occur, the $B$ field is small $(\sim 0.25 \mathrm{G})$ as estimated from the models of Zucca et al. (2014). From our analysis, the location of these $S$ bursts does not meet the condition for ECM to occur. Presently we are not able to draw a conclusion on the emission mechanism of $\mathrm{S}$ bursts and we hope that future imaging observations of $\mathrm{S}$ bursts will constrain these results.

The tied-array beams prove promising in detecting millisecond duration radio bursts with very high frequency and temporal resolution. In addition to this, tied-array beam observations can show the positions of these radio bursts sources. The accuracy in these positions is only limited by the beam size. Ionospheric effects were not significant during this observation. No movement was detected in the $\mathrm{S}$ bursts source position and the Quiet Sun position was constant during the observation. The spatial resolution of tied-array beams can only be increased by increasing the baseline of the LOFAR beam-formed modes (i.e., extend beyond the LOFAR core) which would not have sufficient beams to cover the Sun and is also currently not available as an observational mode. However, at such low frequencies, scattering in the corona may limit the resolution of the beam sizes. Another way to increase the spatial resolution when observing $S$ bursts is to decrease the cadence of LOFAR's interferometric snapshot imaging to $<0.1 \mathrm{~s}$ which is also currently not ideal due to the high data volume rates. This, however, can be compensated by a coarser frequency resolution and it is worth testing in the future. Tied-array beam analysis has been previously applied to Type III radio bursts (Morosan et al. 2014) and it shows a high potential for studying $\mathrm{S}$ bursts and other radio bursts within the LOFAR spectral range.

Acknowledgements. This work has been supported by a Government of Ireland studentship from the Irish Research Council (IRC), the Non-Foundation Scholarship awarded by Trinity College Dublin, the Innovation Academy and the IRC New Foundations. Hamish Reid is supported by a SUPA Advanced Fellowship and an STFC grant ST/L000741/1. We would finally like to acknowledge the LOFAR telescope. LOFAR, the LOw Frequency ARray designed and constructed by ASTRON, has facilities in several countries, that are owned by various parties (each with their own funding sources), and that are collectively operated by the International LOFAR Telescope (ILT) foundation under a joint scientific policy.

\section{References}

Abranin, E. P., Bruck, Y. M., Zakharenko, V. V., \& Konovalenko, A. A. 2001, Exp. Astron., 11, 85

Benz, A. O., Jaeggi, M., \& Zlobec, P. 1982, A\&A, 109, 305

Benz, A. O., Csillaghy, A., \& Aschwanden, M. J. 1996, A\&A, 309, 291

Briand, C., Zaslavsky, A., Maksimovic, M., et al. 2008, A\&A, 490, 339

Dulk, G. A., \& Suzuki, S. 1980, A\&A, 88, 203

Elgaroy, O., \& Sveen, O. P. 1979, Nature, 278, 626

Ellis, G. R. A. 1969, Austral. J. Phys., 22, 177

Ellis, G. R. A. 1982, Austral. J. Phys., 35, 87

Ferris, R. H., Turner, P. J., Hamilton, P. A., \& McCulloch, P. M. 1980, PASA, 4, 26

Gary, D. E., \& Hurford, G. J. 1989, Geophysical Monograph Series 54, (Washington DC: American Geophysical Union), 237

Hess, S., Zarka, P., \& Mottez, F. 2007, Planet. Space Sci., 55, 89

Lemen, J. R., Title, A. M., Akin, D. J., et al. 2012, Sol. Phys., 275, 17

Magdalenić, J., Vršnak, B., Zlobec, P., Hillaris, A., \& Messerotti, M. 2006, ApJ, 642, L77

Mann, G., \& Klassen, A. 2002, in Solar Variability: From Core to Outer Frontiers, ed. A. Wilson, ESA SP, 506, 245

Mann, G., Jansen, F., MacDowall, R. J., Kaiser, M. L., \& Stone, R. G. 1999 A\&A, 348, 614

McConnell, D. 1980, PASA, 4, 64

McConnell, D. 1982, Sol. Phys., 78, 253

McConnell, D. 1983, Sol. Phys., 84, 361

Melnik, V. N., Konovalenko, A. A., Rucker, H. O., et al. 2010, Sol. Phys., 264, 103

Melnik, V. N., Rucker, H. O., Konovalenko, A. A., et al. 2011, in Planetary, Solar and Heliospheric Radio Emissions (PRE VII) Proc., 343

Melrose, D. B. 1991, ARA\&A, 29, 31

Morosan, D. E., Gallagher, P. T., Zucca, P., et al. 2014, A\&A, 568, A67

Newkirk, Jr., G. 1961, ApJ, 133, 983

Saito, K., Poland, A. I., \& Munro, R. H. 1977, Sol. Phys., 55, 121

Stappers, B. W., Hessels, J. W. T., Alexov, A., et al. 2011, A\&A, 530, A80

van Haarlem, M. P., Wise, M. W., Gunst, A. W., et al. 2013, A\&A, 556, A2

Wentzel, D. G. 1984, Sol. Phys., 90, 139

Zaitsev, V. V., \& Zlotnik, E. Y. 1986, Sov. Astron. Lett., 12, 128

Zucca, P., Carley, E. P., Bloomfield, D. S., \& Gallagher, P. T. 2014, A\&A, 564, A47

1 School of Physics, Trinity College Dublin, Dublin 2, Ireland e-mail: morosand@tcd.ie

2 ASTRON, Netherlands Institute for Radio Astronomy, Postbus 2, 7990 AA Dwingeloo, The Netherlands 
3 School of Physics and Astronomy, SUPA, University of Glasgow, Glasgow G12 8QQ, UK

4 Solar-Terrestrial Center of Excellence, SIDC, Royal Observatory of Belgium, Avenue Circulaire 3, 1180 Brussels, Belgium

5 Leibniz-Institut für Astrophysik Potsdam (AIP), An der Sternwarte 16, 14482 Potsdam, Germany

${ }^{6}$ RAL Space, Science and Technology Facilities Council, Rutherford Appleton Laboratory, Harwell Oxford, Oxfordshire, OX11 OQX, UK

7 LESIA, UMR CNRS 8109, Observatoire de Paris, 92195 Meudon, France

8 Institute of Radio Astronomy, 4, Chervonopraporna Str., 61002 Kharkiv, Ukraine

9 Commission for Astronomy, Austrian Academy of Sciences, Schmiedlstrasse 6, 8042 Graz, Austria

10 Swedish Institute of Space Physics, Box 537, 75121 Uppsala, Sweden

11 Space Telescope Science Institute, 3700 San Martin Drive, Baltimore, MD 21218, USA

12 Helmholtz-Zentrum Potsdam, DeutschesGeoForschungsZentrum GFZ, Department 1: Geodesy and Remote Sensing, Telegrafenberg, A17, 14473 Potsdam, Germany

13 Shell Technology Center, Bangalore, 560099 Karnataka, India

14 SRON Netherlands Insitute for Space Research, PO Box 800, 9700 AV Groningen, The Netherlands

15 Kapteyn Astronomical Institute, PO Box 800, 9700 AV Groningen, The Netherlands

16 University of Twente, 7522 NB Enxhede, The Netherlands

17 Harvard-Smithsonian Center for Astrophysics, 60 Garden Street, Cambridge, MA 02138, USA

18 University of Hamburg, Gojenbergsweg 112, 21029 Hamburg, Germany
19 Astrophysics, University of Oxford, Denys Wilkinson Building, Keble Road, Oxford OX1 3RH, UK

20 School of Physics and Astronomy, University of Southampton, Southampton, SO17 1BJ, UK

21 Research School of Astronomy and Astrophysics, Australian National University, Mt Stromlo Obs., via Cotter Road, Weston, A.C.T. 2611, Australia

22 Max Planck Institute for Astrophysics, Karl Schwarzschild Str. 1, 85741 Garching, Germany

23 SmarterVision BV, Oostersingel 5, 9401 JX Assen, The Netherlands

24 Thüringer Landessternwarte, Sternwarte 5, 07778 Tautenburg, Germany

25 Department of Astrophysics/IMAPP, Radboud University Nijmegen, PO Box 9010, 6500 GL Nijmegen, The Netherlands

${ }^{26}$ Leiden Observatory, Leiden University, PO Box 9513, 2300 RA Leiden, The Netherlands

27 LPC2E - Université d'Orléans/CNRS, 3A, Avenue de la Recherche Scientifique, 45071 Orléans Cedex 2, France

28 Station de Radioastronomie de Nançay, Observatoire de Paris CNRS/INSU, USR 704 - Univ. Orléans, OSUC, route de Souesmes, 18330 Nançay, France

29 Anton Pannekoek Institute, University of Amsterdam, Postbus 94249, 1090 GE Amsterdam, The Netherlands

30 Astro Space Center of the Lebedev Physical Institute, Profsoyuznaya str. 84/32, 117997 Moscow, Russia

31 Sodankylä Geophysical Observatory, University of Oulu, Tähteläntie 62, 99600 Sodankylä, Finland

32 STFC Rutherford Appleton Laboratory, Harwell Science and Innovation Campus, Didcot OX11 0QX, UK

33 Center for Information Technology (CIT), University of Groningen, PO Box 11044, 9700 CA Groningen, The Netherlands 